\title{
Performance Evaluation of OFDM based Wi-Max Transceiver
}

\author{
Seema Paliwal \\ Post graduate Student \\ Sagar Institute of Research \& \\ Technology, Bhopal \\ M.Tech. (Digital Communication)
}

\author{
Navneet Kaur \\ Assistant Professor \\ EC Department \\ Sagar Institute of Research \& \\ Technology, Bhopal
}

\author{
Prithviraj Singh Chouhan \\ Assistant Professor \\ EC Department \\ Shri Dadaji Institute of Technology \& \\ Science, Khandwa
}

\begin{abstract}
This Paper describes the simulation \& performance Evaluation of the WIMAX baseband processor. A MATLAB simulation is carried out in order to analyze baseband processing of the transceiver. Orthogonal Frequency Division Multiplexing (OFDM) is applied in this project according to the IEEE 802.16 standard, which allows Transmission of data rates from $6 \mathrm{Mbps}$ up to $54 \mathrm{Mbps}$. Distinct modulation schemes as Binary Phase Shift Keying (BPSK), Quadrate Phase Shift Keying (QPSK) and Quadrature Amplitude modulation (QAM) are used according to differing data Rates for Signal visualization at each block of Transceiver. Simulation is carried to measure Bit Error Rate (BER), Symbol error rate \& to measure Number of data bits, coded bits in OFDM symbol by using different modulation \& Code rate. Pre implementation simulation for WIMAX system is developed to reduce system cost \& to improve effective utilization with limited resources.
\end{abstract}

\section{Keywords}

IEEE 802.16; OFDM; WIMAX; QAM.

\section{INTRODUCTION}

IEEE 802.16 standard, also called WIMAX is the one of the recent additions to the field of communication. The WIMAX standard, approved by IEEE 802.16 workgroup back in 1999 was invented to enhance to the uses of current computer networking. It enables the WIMAX to be easily extended through communications. The technology has proven to be extremely useful in the cases when mobile computers need to connect to existing backbone.The base IEEE 802.16 standard provides asynchronous and connectionless communications at data transfer rates of 6 and $54 \mathrm{Mbps}$. The data rates the standard offers were considered insufficient to incorporate with current WIMAX technology and therefore has never reach into massive crowds. However, as the standard was gradually enhanced (in terms of functionalities and data transfer rate) through document additions for few years, it finally found its way into the IT world. The 802.16 standard was approved in July 1999. It operates at $5 \mathrm{GHz}$ band frequencies and was the first to offer high data transfer rate at 54 Mbps. Jan BENEŠ, Jan PROKOPEC et al [1] investigates impact of different encoding schemes used in Mobile WiMAX to theoretical throughput and deals with efficiency evaluation of these schemes. Sami H. O. Salih, Mamoun M. A. Suliman et al [2] investigates Adaptive Modulation and Coding (AMC) features of the WiMAX and LTE access layer using SDR technologies in Matlab. paper focusing on the physical layer design (i.e. Modulation), here the various used modulation type will be implemented in a single Matlab function that can be called with the appropriate coefficients. A comparison with the hardware approaches will be made in terms of SNR vs. BER relation. Rakesh Kumar Jha, Dr. Upena D. Dalal et al [3] investigates WiMAX physical layer threats jamming and scrambling. The performance of the system was found out to greatly differ with the use of different jamming signals, allowing central areas to be identified, where system development should be focused on. In addition, from the basic theory point of view, rather surprising results were also found. The work should give a clear picture of how the studied WiMAX system performs under jamming as well as without the presence of jamming. The issues related to jamming and jamming reduction techniques are also covered. Jamming and scrambling can destroy communication in the targeted area. Multi-carrier jamming is challenge in WiMAX because WiMAX is having OFDM based physical layer. Simulation approach is main concern here. OPNET MODELER is the software used for the simulation purpose. L.D.MALVIYA et al [4] presents the performance of different variants of transmission control protocols with different modulation schemes when density of mobile nodes changes.

In this paper MATLAB simulation is carried out in order to analyze baseband processing of the transceiver. Orthogonal Frequency Division Multiplexing (OFDM) is applied in this project according to the IEEE 802.16 standard, which allows Transmission of data rates from $6 \mathrm{Mbps}$ up to $54 \mathrm{Mbps}$. Distinct modulation schemes as Binary Phase Shift Keying (BPSK), Quadrate Phase Shift Keying (QPSK) and Quadrature Amplitude modulation (QAM) are used according to differing data Rates for Signal visualization at each block of Transceiver. Simulation is carried to measure Bit Error Rate (BER), Symbol error rate \& to measure Number of data bits, coded bits in OFDM symbol by using different modulation \& Code rate. Pre implementation simulation for WIMAX system is developed to reduce system cost \& to improve effective utilization with limited resources.

\section{PRINCIPLE OF OFDM TRANSMISSION}

Orthogonal Frequency Division Multiplexing (OFDM) is a multiplexing technique that divides a channel with a higher relative data rate into several orthogonal sub-channels with a lower data rate. For high data rate transmissions, the symbol duration Ts is short. Therefore ISI due to multipath propagation distorts the received signal, if the symbol durations are smaller as the maximum delay of the channel. To mitigate this effect a narrowband channel is needed, but for high data rates a broadband channel is needed. 


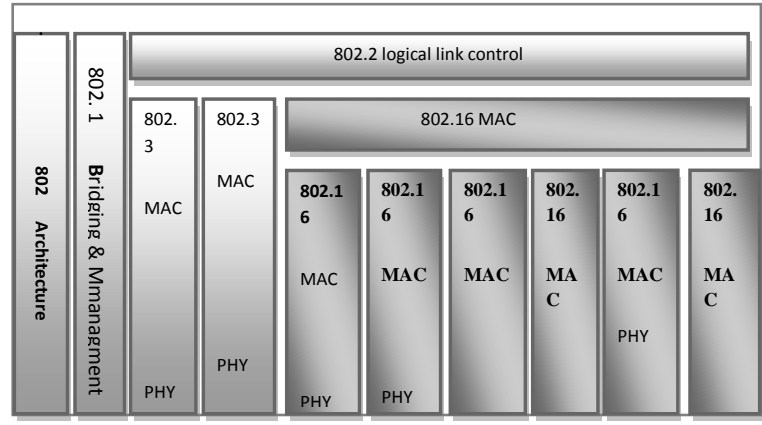

Fig 1 -IEEE 802.16 architecture

To overcome this problem the total bandwidth can be split into several Parallel narrowband subcarriers. Thus a block of $\mathrm{N}$ serial data symbols with Duration Ts is converted into a block of $\mathrm{N}$ parallel data symbols, each with Duration $\mathrm{T}=$ $\mathrm{N} \times \mathrm{Ts}$. The aim is that the new symbol duration of each subcarrier is larger than the maximum delay of the channel, $\mathrm{T}$ $>\mathrm{T}$ max. With many low data rate subcarriers at the same time, a higher data rate is achieved. In order to create the OFDM symbol a serial to parallel block is used to convert $\mathrm{N}$ serial data symbols into $\mathrm{N}$ parallel data symbols. Then each parallel data symbol is modulated with a different orthogonal frequency subcarriers, and added to an OFDM symbol

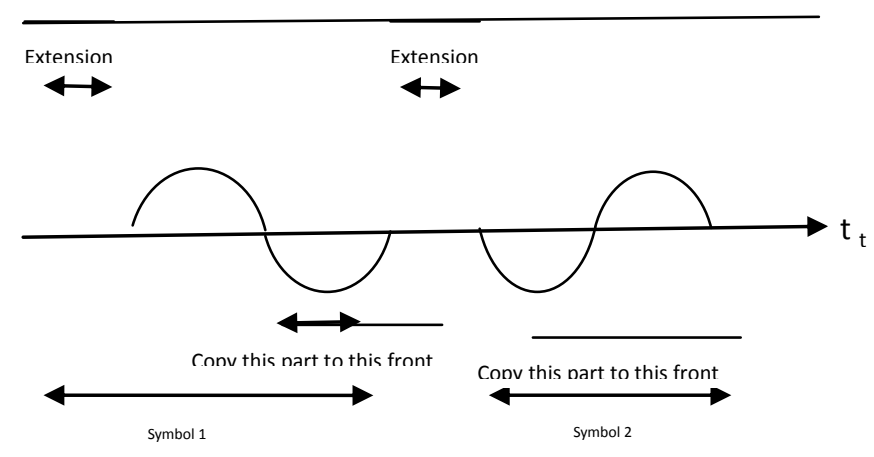

Fig 2-Cyclic prefix Insertion

The format for the PPDU including the OFDM PLCP preamble, OFDM PLCP header, PSDU, tail bits, and pad bits is shown in Fig 3 the PLCP header contains the following fields: LENGTH, RATE, a reserved bit, an even parity bit, and the SERVICE field. In terms of modulation, the LENGTH, RATE, reserved bit, and parity bit (with 6 "zero" tail bits appended) constitute a separate single OFDM symbol, denoted SIGNAL, which is transmitted with the most robust combination of BPSK modulation and a coding rate of $\mathrm{R}=$ $1 / 2$. The SERVICE field of the PLCP header and the PSDU (with 6 "zero" tail bits and pad bits appended), denoted as DATA, are transmitted at the data rate described in the RATE field and may constitute multiple OFDM symbols.

\begin{tabular}{|l|l|l|l|l|l|l|l|l|}
\hline $\begin{array}{l}\text { RAT } \\
\mathrm{E} \\
\text { aL:-2. }\end{array}$ & $\begin{array}{l}\text { Reserve } \\
\mathrm{d} 1 \\
\text { bits }\end{array}$ & $\begin{array}{l}\text { Length } \\
12 \text { bits }\end{array}$ & $\begin{array}{l}\text { Parity } \\
1 \text { bits }\end{array}$ & $\begin{array}{l}\text { Tail } 6 \\
\text { bits }\end{array}$ & $\begin{array}{l}\text { Service } \\
16 \text { bits }\end{array}$ & PSDU & $\begin{array}{l}\text { Tail } 6 \\
\text { bits }\end{array}$ & $\begin{array}{l}\text { Pad } \\
\text { Bits }\end{array}$ \\
\hline & & & & \\
\hline
\end{tabular}

Fig 3- PPDU frame format

The tail bits in the SIGNAL symbol enable decoding of the RATE and LENGTH fields immediately after the reception of the tail bits. The RATE and LENGTH are required for decoding the DATA part of the packet. In addition, the CCA mechanism can be augmented by predicting the duration of the packet from the contents of the RATE and LENGTH fields, even if the data rate is not supported by the station.

\section{KEY OFDM PARAMETERS}

This clause specifies an orthogonal frequency division multiplexing (OFDM) system and the additions that have to be made to the base standard to accommodate the OFDM PHY. The radio frequency WIMAX system is initially aimed for the 5.15-5.25, 5.25-5.35 and 5.725-5.825 GHz. The OFDM system provides WIMAX with data payload communication capabilities of $6,9,12,18,24,36,48$, and 54 $\mathrm{Mbit} / \mathrm{s}$. The support of transmitting and receiving at data rates of 6,12 , and $24 \mathrm{Mbit} / \mathrm{s}$ is mandatory. The system uses 52 subcarriers that are modulated using binary or Quadrature phase shift keying (BPSK/QPSK), 16-quadrature amplitude modulation (QAM), or 64-QAM.Where Forward error correction coding (convolutional coding) is used with a coding rate of $1 / 2,2 / 3$, or $3 / 4$.The transmitted signals will be described in a complex baseband signal notation.

The actual transmitted signal is related to the complex baseband signal by the following relation:

$$
r(R F)\langle t\rangle=\left\{r\langle t\rangle \exp \left\langle j 2 \Pi^{*} f c t\right\rangle\right\}
$$

Where $\operatorname{Re}($.$) represents the real part of a complex variable;$ Fc denotes the carrier center frequency. The transmitted baseband signal is composed of contributions from several OFDM symbols.

\section{$r$ Packet $(t)=r$ Preamble $(t)+r$ Signal $(t-t$ signal $)+r$ Data $(t-t d a t a)$}

The time offsets tSUBFRAME determine the starting time of the corresponding sub frame; tSIGNAL is equal to $16 \mu \mathrm{s}$, and data is equal to $20 \mu \mathrm{s}$.

The parameters $\Delta \mathrm{F}$ and NST are described in Table 1 the resulting waveform is periodic with a period of TFFT $=1 / \Delta \mathrm{F}$. Shifting the time by TGUARD creates the "circular prefix" used in OFDM to avoid ISI from the previous frame. Three kinds of GUARD are defined: for the short training sequence $(=0 \mu \mathrm{s})$, for the long training sequence (= TGI2), and for data OFDM symbols (= TGI). (Refer to Table 1$)$ The boundaries of the sub frame are set by a multiplication by a time- 
windowing function, wTSUBFRAME(t), which is defined as a rectangular pulse, $w \mathrm{~T}(\mathrm{t})$, of duration $\mathrm{T}$, accepting the value TSUBFRAME. The time-windowing function, wT(t), depending on the value of the duration parameter, $\mathrm{T}$, may extend over more than one period, TFFT.

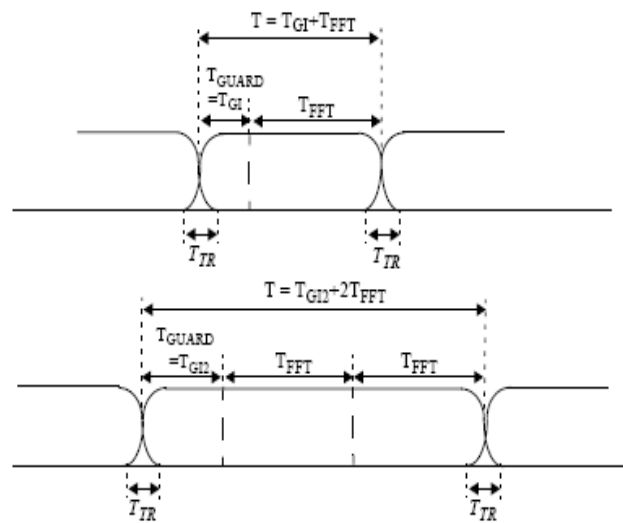

Figure 4-Illustration of OFDM frame with cyclic extension and windowing for (a) Single Reception or (b) two receptions of the FFT period

In particular, window functions that extend over multiple periods of the Fast Fourier Transform (FFT) are utilized in the definition of the preamble. The possibility of extending the windowing function over more than one period, TFFT, and additionally shows smoothed transitions by application of a windowing function. In particular, window functions that extend over multiple periods of the FFT are utilized in the definition of the preamble.

Table 1 is the list of timing parameters associated with the OFDM PLCP (For BPSK Signal)

\begin{tabular}{|c|c|}
\hline Parameter & Value \\
\hline $\mathrm{N}_{\mathrm{SD}}$ : No of Data Subcarriers & 48 \\
\hline $\mathrm{N}_{\mathrm{SP}}$ :No of pilot Subcarriers & 4 \\
\hline $\mathrm{N}_{\mathrm{ST}}$ : No of total Subcarriers & $52\left(\mathrm{~N}_{\mathrm{SD}+} \mathrm{N}_{\mathrm{SP}}\right)$ \\
\hline$\Delta \mathrm{f}:$ Subcarrier Frequency Spacing & $\begin{array}{l}0.3125 \mathrm{MHz}(20 \mathrm{MHz} \\
/ 64)\end{array}$ \\
\hline $\mathrm{T}_{\mathrm{FFT}}:$ IFFT/FFT Period & $3.2 \mu \mathrm{Sec}$ \\
\hline $\begin{array}{l}\text { TPREAMABLE :PCLP preamble } \\
\text { duration }\end{array}$ & $\begin{array}{l}16 \mu \mathrm{Sec}\left(\mathrm{T}_{\mathrm{SHORT}+}\right. \\
\left.\mathrm{T}_{\text {LONG }}\right)\end{array}$ \\
\hline $\mathrm{T}_{\text {SIGNAL: }}$ : Duration of OFDM symbol & $4.0 \mu \operatorname{Sec}\left(\mathrm{T}_{\mathrm{GI}+} \mathrm{T}_{\mathrm{FFT}}\right)$ \\
\hline $\mathrm{T}_{\mathrm{GI}}$ :GI duration & $0.8 \mu \mathrm{Sec}\left(\mathrm{T}_{\mathrm{FFT} /} / 4\right)$ \\
\hline $\mathrm{T}_{\mathrm{GI} 2}$ :Training Symbol GI duration & $1.6 \mu \mathrm{Sec}\left(\mathrm{T}_{\mathrm{FFT} / 2}\right)$ \\
\hline $\mathrm{T}_{\text {SYM }}$ :Symbol Duration & $4.0 \mu \operatorname{Sec}\left(\mathrm{T}_{\mathrm{GI}}+\mathrm{T}_{\mathrm{FFT}}\right)$ \\
\hline $\begin{array}{l}T_{\text {SHORT }}: \text { Short sequence Training } \\
\text { Duration }\end{array}$ & $8 \mu \operatorname{Sec}\left(10^{*} \mathrm{~T}_{\mathrm{FFT} /} 4\right)$ \\
\hline $\begin{array}{l}T_{\text {LONG }} \text { : Long sequence Training } \\
\text { Duration }\end{array}$ & $8 \mu \operatorname{Sec}\left(2 * \mathrm{~T}_{\mathrm{FFT}}+\mathrm{T}_{\mathrm{GI} 2}\right)$ \\
\hline
\end{tabular}

A short OFDM training symbol consists of 12 subcarriers, which are modulated by the elements of the sequence $\mathrm{S}$, given by

$\mathrm{S}-26,26=\sqrt{ }(13 / 6) \times\{0,0,1+\mathrm{j}, 0,0,0,-1-\mathrm{j}, 0,0,0,1+\mathrm{j}, 0,0$, $0,-1-\mathrm{j}, 0,0,0,-1-\mathrm{j}, 0,0,0,1+\mathrm{j}, 0,0,0,0,0,0,0,-1-\mathrm{j}, 0,0$, $0,-1-\mathrm{j}, 0,0,0,1+\mathrm{j}, 0,0,0,1+\mathrm{j}, 0,0,0,1+\mathrm{j}, 0,0,0,1+\mathrm{j}, 0,0\}$

The multiplication by a factor of $\sqrt{ }(13 / 6)$ is in order to normalize the average power of the resulting OFDM symbol, which utilizes 12 out of 52 subcarriers.

A long OFDM training symbol consists of 53 subcarriers, which are modulated by the elements of the sequence $\mathrm{L}$, given by

$\mathrm{L}-26,26=$

$\{1,1,-1,-1,1,1,-1,1,-1,1,1,1,1,1,1,-1,-1,1,1,-1,1$, $-1,1,1,1,1,0,1,-1,-1,1,1,-1,1,-1,1,-1,-1,-1,-1,-1$, $1,1,-1,-1,1,-1,1,-1,1,1,1,1\}$

The modulation parameters dependent on the data rate used shall be set according to Table 2

Table 2 -Rate Dependent parameters

\begin{tabular}{|l|l|l|l|l|l|}
\hline $\begin{array}{l}\text { Data } \\
\text { rate }\end{array}$ & Modulation & $\begin{array}{l}\text { Coding } \\
\text { rate (R) }\end{array}$ & NBPSC & NCBPS & NDBPS \\
\hline 6 & BPSK & $1 / 2$ & 1 & 48 & 24 \\
\hline 9 & BPSK & $3 / 4$ & 1 & 48 & 36 \\
\hline 12 & QPSK & $1 / 2$ & 2 & 96 & 48 \\
\hline 18 & QPSK & $3 / 4$ & 2 & 96 & 72 \\
\hline 24 & 16 QAM & $1 / 2$ & 4 & 192 & 96 \\
\hline 36 & 16 QAM & $3 / 4$ & 4 & 192 & 144 \\
\hline 48 & 64 QAM & $1 / 2$ & 6 & 288 & 192 \\
\hline 54 & 64 QAM & $3 / 4$ & 6 & 288 & 216 \\
\hline
\end{tabular}

\section{WIMAX BASEBAND PROCESSOR}

Basic WIMAX Baseband processor as shown in Fig 5 consists of transmitter which is comprised of a codec, a modulator and IFFT blocks. The receiver is comprised of a synchronization block, a FFT, an equalizer, a demodulator and a Viterbi decoder in the codec. The baseband processor consists of a codec based on the Viterbi decoding algorithm and the modem including an IFFT/FFT, synchronization block and equalizer

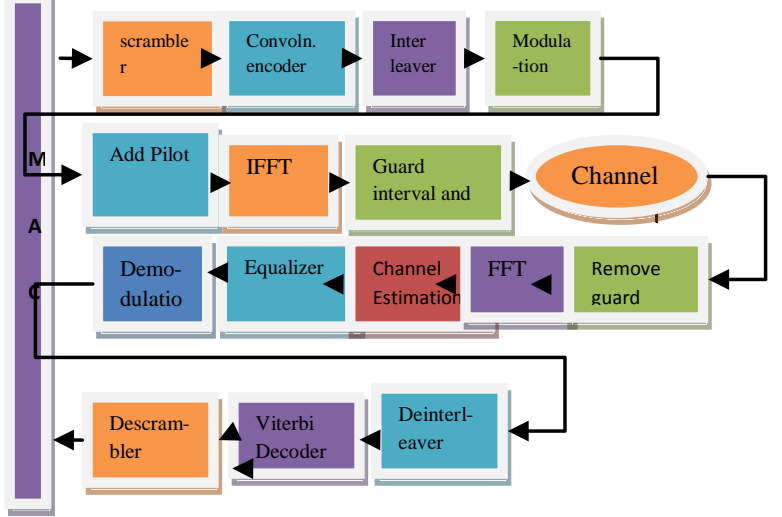

Fig 5- Basic WIMAX baseband processor 
The BER calculation after demodulator, and before the Viterbi decoder, depends on the type of modulation and the channel type. Due to error correction mechanisms of the convolutional codes, the BER before the decoder is not the same as the BER after the decoder. For deriving the latter, we need to have knowledge about the used convolutional code. The first section is dedicated to introducing the methods used to derive the BER before the Viterbi decoder, and after the demodulator, and the second section treats the BER calculation methods after the Viterbi decoder.BER after Modulator, Before Decoder.

In every chuck in the packet, where $\mathrm{Ni}$ and Signal level are constant, we calculate

$\frac{E_{b}}{N_{o}}(k, t)=\operatorname{SNIR}(k, t) \frac{B_{t}}{R_{b}(k, t)}$

Where $\mathrm{Eb}$ is energy per bit, $\mathrm{NO}$ is the noise power density, $\mathrm{Bt}$ is the bandwidth of the signal $(20 \mathrm{MHz}$ in $802.11 \mathrm{a})$ and $\mathrm{Rb}(\mathrm{k}, \mathrm{t})$ is the bit rate of transmission for packet $\mathrm{k}$ at time $\mathrm{t}$.

\subsection{Types of Channels for BER}

The $\mathrm{Q}$ function, the Error Function, erf (), and the Complementary Error Function, erfc (), are used in the following formulas. Here are the basic definitions and relations:

$$
Q(u)=\int_{M}^{\infty} \frac{\exp \left(-t^{2} / 2\right)}{\sqrt{2 \Pi}} d t
$$

The relation between $\mathrm{Q}$ function and erfc function;

$$
Q(x)=.5 \times \operatorname{erfc}\left(\frac{x}{\sqrt{2}}\right)
$$

The relation between Bit SNR, Symbol SNR, Symbol error probability $\mathrm{Ps}$, and Bit error probability $\mathrm{Pb}$ is given by

$$
\begin{aligned}
S N R_{S} & =\log _{2}^{M} \times S N R_{b} \\
P_{S} & =\log _{2}^{M} \times P_{b}
\end{aligned}
$$

The above approximate conversions typically assume that the symbol energy is divided equally among all bits, and that Gray encoding is used so that at reasonable SNRs, one symbol error corresponds to exactly one bit error.

\subsection{BER after Viterbi Decoder}

The Bit Error Rate, as mentioned in the introduction, is not equal before and after the Viterbi decoder, due to error correction mechanisms provided by convolutional codes. The procedure to derive the BER after the decoder is as Follows.

As the first step, we calculate the probability of selecting an incorrect path by the Viterbi decoder which is in distance $\mathrm{k}$ from the all-zero path (due to linear Characteristics of the encoder, without loss of generality, we consider that the Sent data were a train of zero bits). The probability $\mathrm{Pk}$ is derived as follows:

$$
\begin{gathered}
p(k)=\sum_{n=\frac{k+1}{2}}^{k}\left(\begin{array}{l}
k \\
n
\end{array}\right) p^{n}(1-p)^{k-n} \\
p(k)=\sum_{n=\frac{k+1}{2}}^{k}\left(\begin{array}{l}
k \\
n
\end{array}\right) p^{n}(1-p)^{k-n}+\frac{1}{2}\left(\begin{array}{l}
k \\
\frac{1}{2} k
\end{array}\right) p^{\frac{k}{2}}(1-p)^{\frac{k}{2}}
\end{gathered}
$$

Where $\mathrm{p}$ is the BER before decoder, for calculating BER for each chunk of bits in the packet, we calculate the first 10 elements of $P_{k}$, multiply each by the corresponding Ck1 value and sum over the result of multiplications. This sum is the BER after decoder for the bits in the given chuck. Here is the formula to calculate BER from $\mathrm{Ck}$ and $\mathrm{Pk}$ values:

$$
B E R<\frac{1}{P u n c} \sum_{k=d_{\text {free }}}^{\infty} C_{k} P_{k}
$$

Punc, in the above formula, is the puncturing period of the convolutional code.

\section{AWGN Channel}

\begin{tabular}{|c|c|}
\hline $\begin{array}{c}\text { BPSK } \\
\text { Modulation }\end{array}$ & $P_{b}=Q\left(\sqrt{2 \gamma_{b}}\right)$ \\
\hline $\begin{array}{c}\text { QPSK } \\
\text { Modulation }\end{array}$ & $p_{s}(E)=2 Q\left(\sqrt{\frac{E_{s}}{N_{o}}}\right)-Q^{2}\left(\sqrt{\frac{E_{s}}{N o}}\right)$ \\
\hline M ary QAM & $P_{s}=1-\left(1-\frac{2(\sqrt{M}-1)}{\sqrt{M}} \times Q\left(\sqrt{\frac{3 \bar{\gamma}}{M-1}}\right)\right)^{2}$ \\
\hline
\end{tabular}

\section{SIMULATION RESULTS}

According to Table 2, select any one modulation scheme with specified code rate $\&$ data rate to visualize signals at different blocks baseband processor. Following graphical window shows results in processor. MATLAB simulation results for 64 Ary QAM with data rate 54Mbits/sec, coding rate $3 / 4$ is carried out to show Maximum no of data bits in one OFDM symbol.

Data rate, Modulation- 54 Mbps, 64 QAM

Coding rate (R) - 3/4

NBPSC -6 


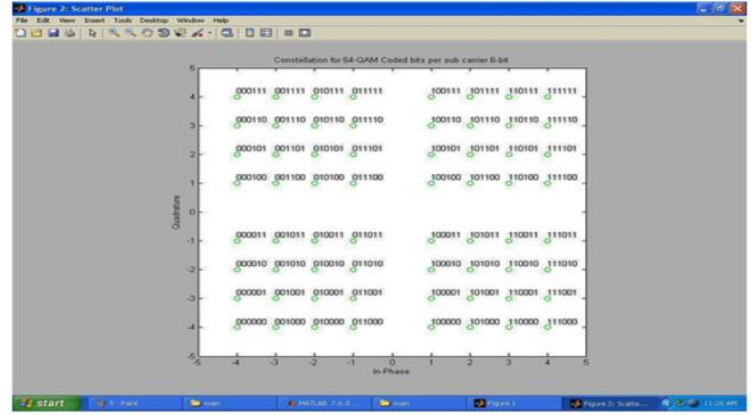

Fig.6 -Constellation for coded bits per subcarrier bit.

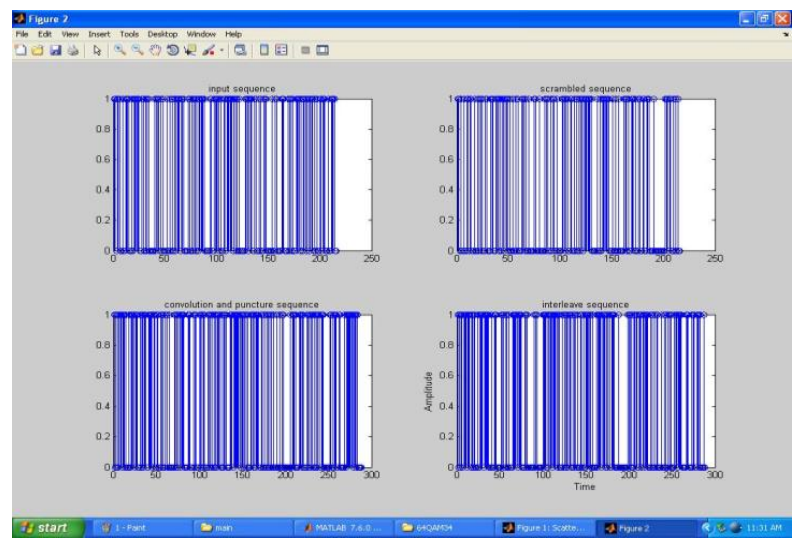

Fig.7 -Input Sequence, Scrambled sequence, convolution $\&$ puncture sequence, Interleave sequence at Transmitter side of Baseband processor.

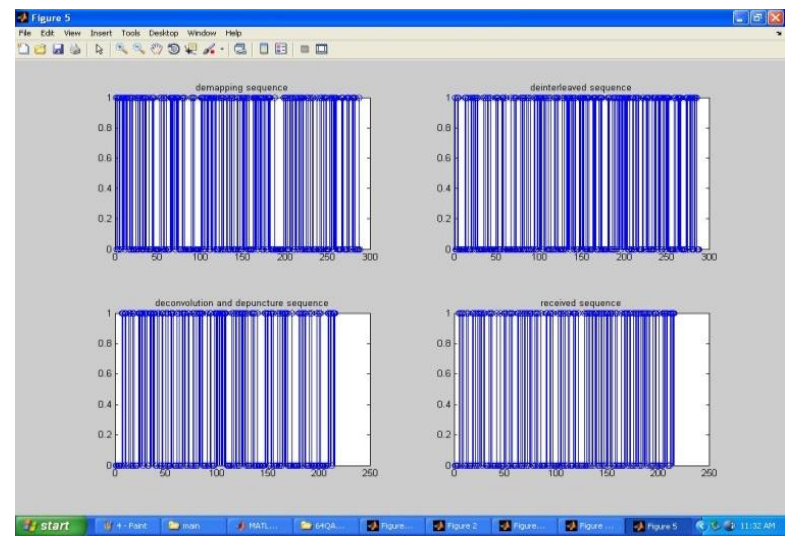

Fig..8-scatter plot of 64 QAM

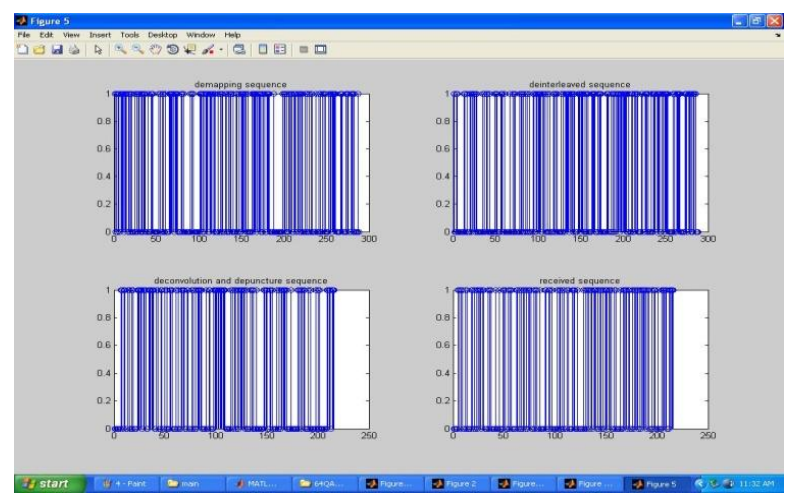

Fig..9-Demapping, Deinterleaved, Deconvolve \& depunture, Received sequence at receiver side of baseband processor.
No of coded bits per Symbol (NCBPS) - 288

No of Data bits per Symbol NDBPS-216

\section{SYMBOL ERROR PROBABILITY.}

The distance between the constellation points for 16QAM modulation is

$d_{16_{q a m}}=2 \sqrt{\frac{E s}{10}}$

Comparing both

$$
\text { ratio }=\frac{d_{16 q a m}}{d_{16 p s k}}=\frac{2 \sqrt{\frac{E s}{10}}}{2 \sqrt{E s} \operatorname{Sin}\left(\frac{\Pi}{16}\right)}=1.62 \mathrm{The}
$$

distance between the constellation points for 16QAM modulation is around 1.6x the value for 16PSK modulation. Expressing in dB's, this comes to

$$
\text { ratio }_{d B}=20 \log (1.62)=4.19 \mathrm{~dB}
$$

More the distance between the constellations, lesser is the chance of a constellation point getting decoded incorrectly. This implies that for the same symbol error rate, 16QAM modulation requires only $4.19 \mathrm{~dB}$ lesser signal to noise ratio, when compared with 16PSK modulation.

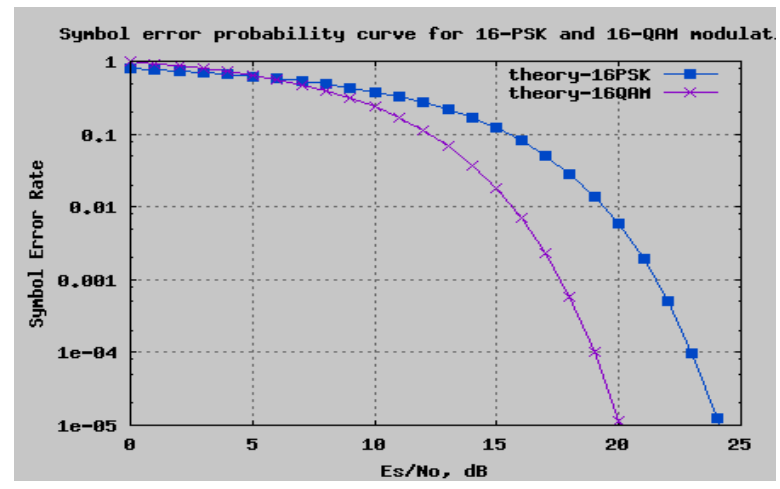

Fig 10- Symbol Error Probability Vs SNR

\section{FADING AND PERFORMANCE IMPROVEMENT IN WIMAX SYSTEM .}

The term fading is used to describe the rapid fluctuations of the amplitudes, phases, or multipath delays of a signal over a short period of time or distance. It is caused by interference between multiple versions of the transmitted signal which arrive at the receiver at slightly different times. Hence, the resulting signal at the receiver may have a wide-varying amplitude and phase. In short, the effects of multipath are rapid changes in signal strength over a small travel distance or time interval, random frequency modulation due to varying Doppler shifts on different multipath signals and time dispersion caused by multipath propagation delays. The multipath components combine vectorially at the receiver which causes the signal to distort, to fade or even to strengthen at times [9].

The presence of reflecting objects and scatterers in the channel causes a constant change in the propagation environment. This changing environment alters the signal 
energy in amplitude, phase, and time, and as a result, multipath propagation occurs causing signal fading. The transmitted signal arrives at the receiver via multiple propagation paths, each of which has an associated time delay. Because the received signal is spread in time due to the multipath scatterers at different delays, the channel is said to be time dispersive. The difference between the largest and the smallest among these delays defines the maximum delay spread. On the other hand, when the receiver and the transmitter are in relative motion, the received signal is subject to a constant frequency shift, called the Doppler shift. Therefore, as it occurs in the time domain, the Doppler spread is defined as the difference between the largest and the smallest among these frequency shifts,

$$
f d=f M \cos \phi
$$
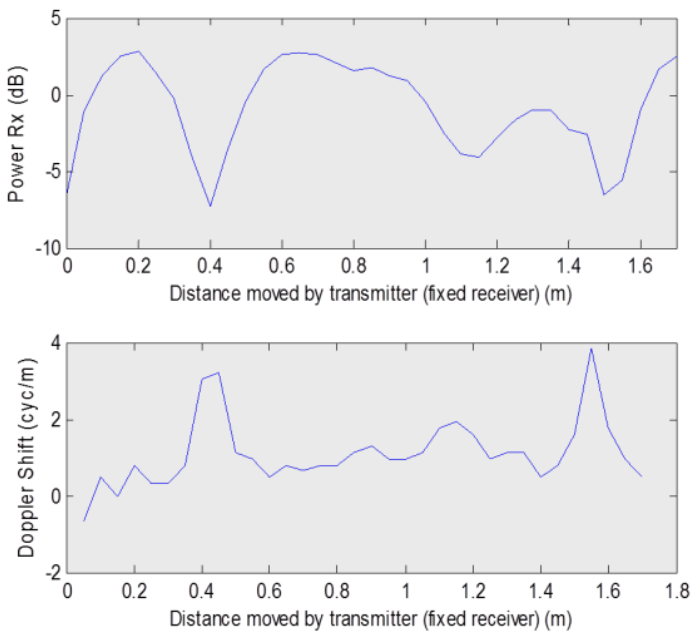

Fig 11- Response of Doppler effect (moving host)

\section{CONCLUSION AND FUTURE SCOPE}

This paper presents effect of Modulation method, code rate; data rate on WIMAX baseband processor which enforces to use 64 QAM with $54 \mathrm{Mbps}$ data rate \& 3/4 code rate. Performance Evaluation is carried by Measuring Symbol error probability for QAM technique. The Doppler Shift \& Received Power between Access point and Baseband Transceiver will gives the exact position to locate AP, where Received power in an environment is plotted over area by using pre implementation model build in MATLAB will limits the number of access points, system cost results effective utilization of access points.

The implemented PHY layer model still needs some improvement. The channel estimator can be implemented to obtain a depiction of the channel state to combat the effects of the channel using an equalizer.

\section{ACKNOWLEDEMENT}

Our thanks to the experts my guide Mrs.Navneet kaur who have contributes towards this paper also special thanks to SDITS college of Khandwa which provide MATLAB for this work.

\section{REFERENCES}

[1] Jan BENEŠ, Jan PROKOPEC" Efficiency of Encoding Schemes in Mobile WiMAX"

[2] Sami H. O. Salih, Mamoun M. A. Suliman" Implementation of Adaptive Modulation and Coding Technique using" International Journal of Scientific \& Engineering Research Volume 2, Issue 5, May-2011

[3] Rakesh Kumar Jha, Dr. Upena D. Dalal "A Performance Comparison With Modulation Schemes In WiMAX Physical Layer Security Aspect" International Journal of Computer Applications (0975 - 8887) Volume 6- No.8, September 2010

[4] L.D.MALVIYA" Simulation based comparison of different Modulation schemes for Mobile WiMAX using TCP and its Variants" First International Conference on Emerging Trends in Engineering and Technology

[5] IEEE 802.16Task Group a, Part 11: WIMAXMedium Access Control (MAC) and Physical Layer (PHY) Specifications: High-speed Physical Layer in the $5 \mathrm{GHz}$ Band, 1999.

.[6] IEEE paper "Implementation of IEEE 802.16 WIMAX Baseband Processor", Je-Hoon Lee, Young-Il Lim, and Kyoung Rok Cho published on December 2008

[7] Vinit Grewal, Ajay K Sharma" On Performance Evaluation of Different QoS Mechanisms and AMC scheme for an IEEE 802.16 based WiMAX Network" International Journal of Computer Applications (0975 8887) Volume 6-No.7, September 2010

[8] Bhalchandra B. Godbole, Dilip S. Aldar "Performance Improvement by Changing Modulation Methods for Software Defined Radios" (IJACSA) International Journal of Advanced Computer Science and Applications, Vol. 1, No. 6, December 2010

[9] Dariush Mohammad Soleymani, Vahid Tabataba Vakili" Improvement of System Capacity using Different Frequency Reuse and HARQ and AMC in IEEE 802.16 OFDMA Networks"

[10] IEEE Communications Society Qingwen Liu, Shengli Zhou and Georgios B. Giannakis, "TCP performance in wireless access with adaptive modulation and coding 2004. 